\title{
Agent Affecting Musculoskeletal System
}

National Cancer Institute

\section{Source}

National Cancer Institute. Agent Affecting Musculoskeletal System. NCI Thesaurus. Code C78281.

Any agent that exerts an effect on muscle, bones and joints. 